\title{
RELATIVISTIC CORRECTIONS TO ATOMIC ENERGY LEVELS
}

\author{
MICHAEL JONES \\ Dept. of Physics, University College London, England
}

\begin{abstract}
The relativistic effects on bound levels of atomic systems with particular application to systems of astrophysical importance are investigated by including the most important contributions to the low $Z$ Pauli Approximation for the Hamiltonian. This work is an extension of a general purpose automatic computer program for calculating non-relativistic energy levels of complex atoms, described by Eissner and Nussbaumer (1969). The program should give fairly good intermediate coupling wave functions and it may thus be used as a basis for the calculation of intermediate coupling collision strenghts and oscillator strenghts. Numerical results are displayed for the Sodium I sequence.
\end{abstract}

A general automatic computer program for the calculation of the structure of complex atoms has been described by Eissner and Nussbaumer (1969). However, their program finds the non-relativistic solution of the structure problem, and as they show this is often unsatisfactory for the higher stages of ionization of atoms of medium nuclear charge $Z$. This program has now been extended to include the principal relativistic effects. Our approach has been to take the non-relativistic wavefunctions given by Eissner and Nussbaumer as a basis set for the application of perturbation theory to the low $Z$ Pauli Hamiltonian, which is the approximation to the relativistic Hamiltonian in the limit of fairly low electron velocities. Thus we can write:

$$
H=H_{n r}+H_{\mathrm{re} 1}
$$

where $H_{n r}$ makes the largest contribution, and $H_{\text {rel }}$ is a correction term. $H_{\text {rel }}$ may be expressed as a sum of one-electron and two-electron operators thus:

$$
H_{\mathrm{rel}}=\sum_{i=1}^{N} f_{i}+\sum_{i>j=1}^{N} g_{i j}
$$

where the $f_{i}$ and $g_{i j}$ are defined by Jones (1970).

In our approximation we have included all the one-electron operators, which can be shown to make the largest contributions to the energy levels for the cases in which we are interested, and one of the two-electron operators namely the spin-other-orbit operator. This latter has been included because it improves the accuracy of the spinorbit parameters, the parameters which to a large part determine the fine structure splitting (Blume and Watson, 1962).

The $Z$-dependence of the energy levels has been considerably improved by the inclusion of these operators in the Hamiltonian. The behaviour of the energy levels along the isoelectronic sequence has been studied following the methods of Layzer (1959). He stated that an energy level $E^{i}$ of a complex atom may be expanded thus:

$$
E^{i}=E_{0}^{i} Z^{2}+E_{1}^{i} Z+E_{2}+\cdots
$$


where $E$ is the same for all levels $E^{i}$ belonging to the same complex, defined by a set of principal quantum numbers and a given parity. The difference between two levels $E^{i}$ and $E^{j}$ belonging to the same complex is:

$$
\Delta E^{i j}(Z)=E^{i}-E^{j}=\left(E_{1}^{i}-E_{1}^{j}\right) Z+E_{2}^{i}-E_{2}^{j}+\cdots
$$

$\delta E(Z)$ is defined by

$$
\delta E(Z)=\Delta E(Z)-\Delta E(Z-1)
$$

Hence

$$
\delta E(Z)=E_{1}^{i}-E_{1}^{j}+O\left(Z^{0}\right)
$$

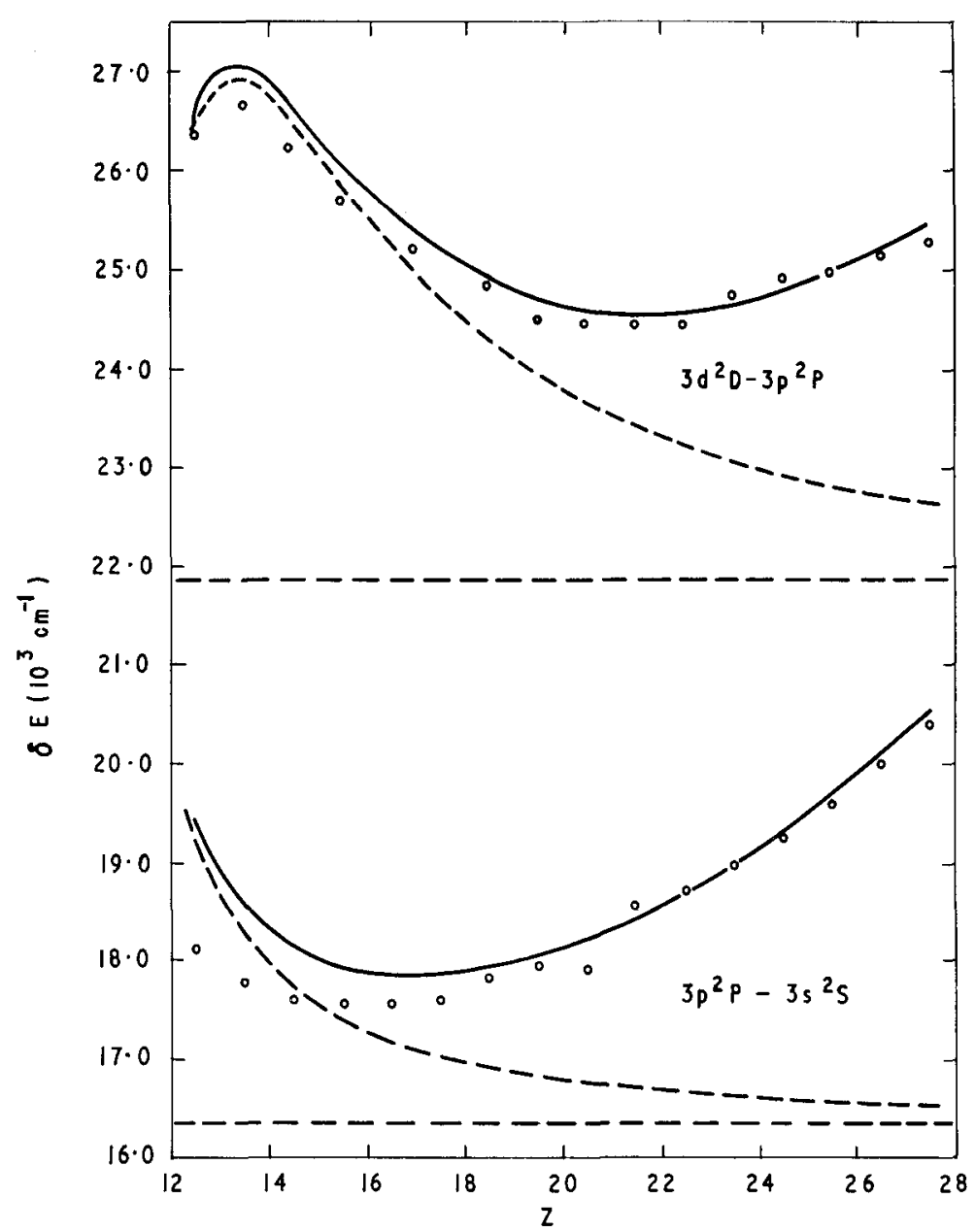

Fig. 1. Sodium I isoelectronic sequence, $\delta E$ vs $Z$ for the transitions $3 p^{2} P-3 s^{2} S$ and $3 d^{2} D-3 p^{2} P$ OExpriment -..- Non-relativistic calculation Relativistic calculation---Godfredsen (1966). 


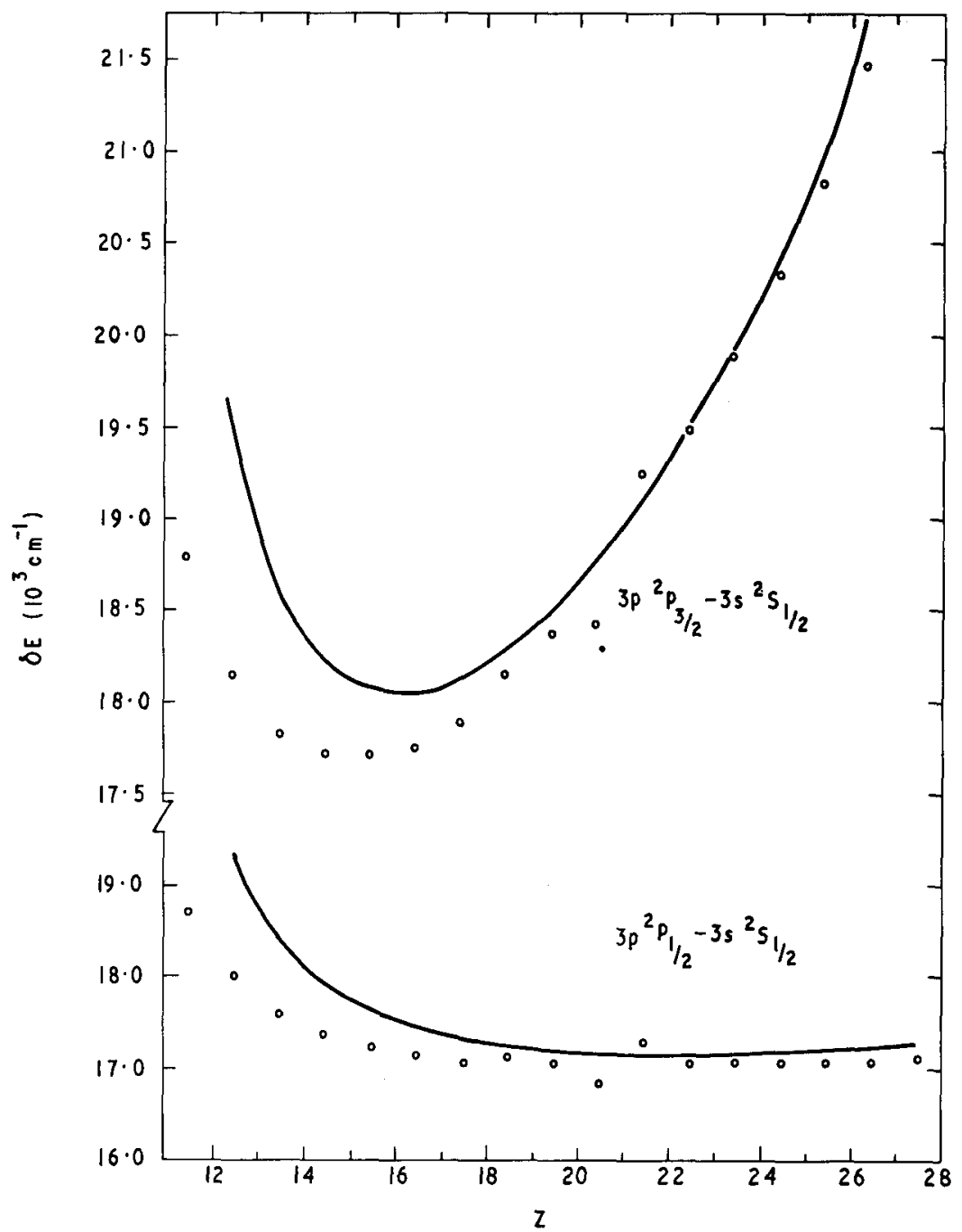

Fig. 2. Sodium I sequence, $\delta E$ vs $Z$ for the transitions $3 p^{2} P_{1 / 2}-3 s^{2} S_{1 / 2}$ and $3 p^{2} P_{3 / 2}-3 s^{2} S_{1 / 2}$ Experiment - - Relativistic calculations. 
Hence in the limit of large $Z, \delta E(Z)$ should tend to a constant, provided no relativistic effects contribute.

In Figure 1, $\delta E$ is plotted against $Z$ for the transitions $3 p^{2} P-{ }^{3} s^{2} S$ and $3 d^{2} D-3 s^{2} S$ in the sodium I sequence. It can be seen that although Eissner and Nussbaumer's results tend to a constant, this does not correspond to the behaviour of the experimental data, which is fitted quite well by the present work. In Figure 2, $\delta E$ has been plotted for the fine structure transitions $3 p^{2} P_{3 / 2}-3 s^{2} S_{1 / 2}$ and $3 p{ }^{2} P_{1 / 2}-3 s^{2} S_{1 / 2}$. In Figures 3 and 4, the spin-orbit parameters deduced from experiment are compared with the calculations of other workers and with the present work.

Calculations have also been done for the $\mathrm{Mg} I$ and $\mathrm{FI}_{\mathrm{I}}$ sequences, where similar improvements in the $Z$-dependence of the energy levels have been obtained (Jones, 1970).

This program has now been applied to the calculation of oscillator strengths and collision strengths in intermediate coupling.

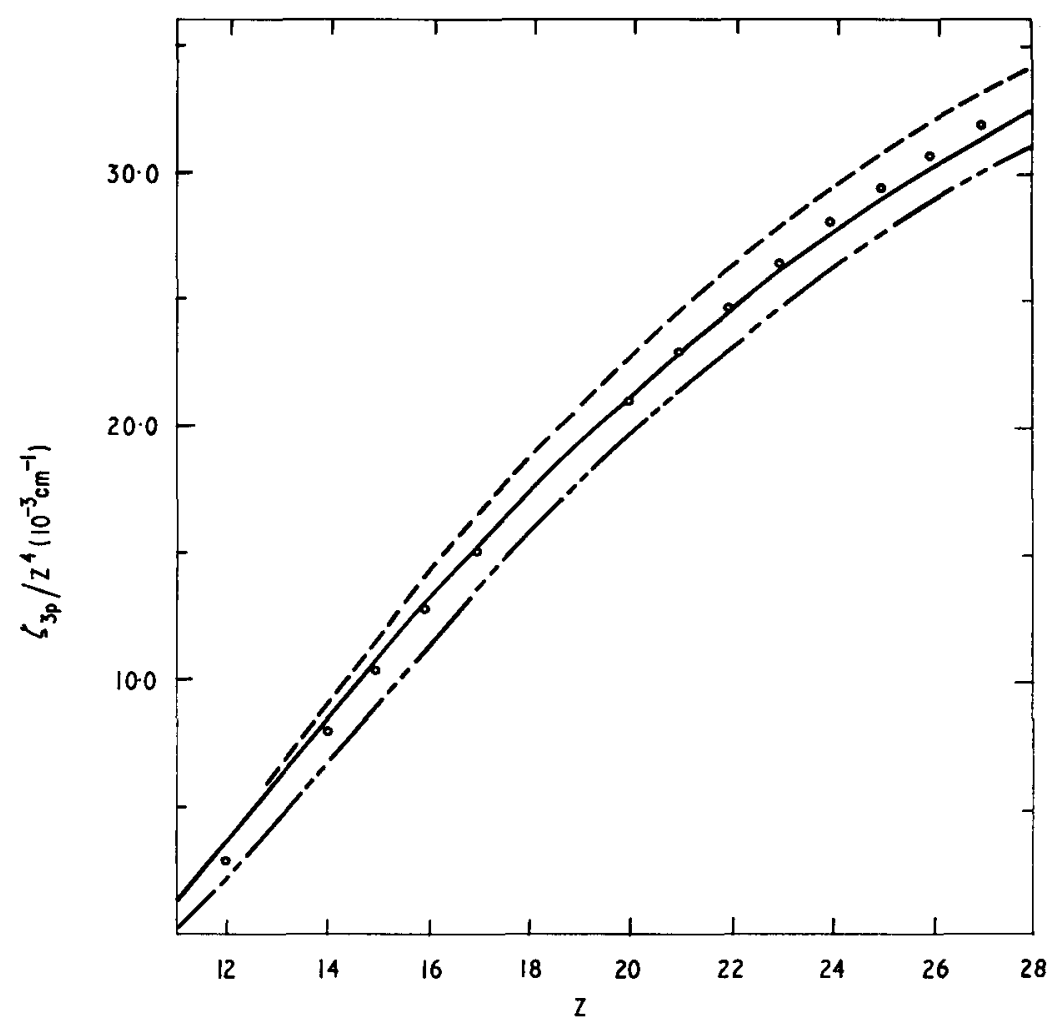

Fig. 3. Sodium sequence $\zeta 3_{p} \mid \mathbf{Z}^{4}$ vs $Z \bigcirc$ Experiment -.-.-- Condon and Odabasi (1966), --- Froese (1967), Present calculation. 


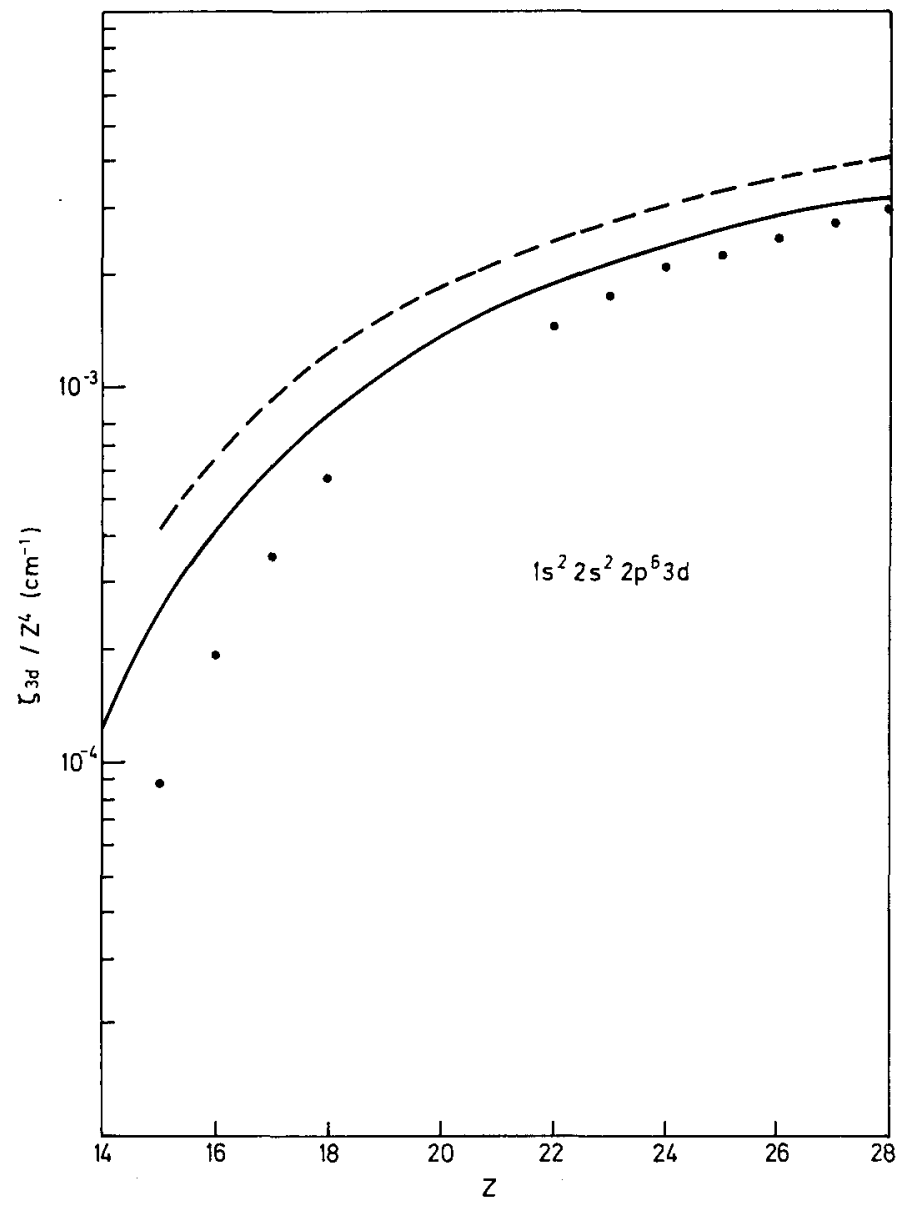

Fig. 4. Sodium I sequence $\zeta_{3 d} \mid Z^{4}$ vs $Z$ Experiment, ...... Condon and Odabasi (1966). Present calculation. Froese's calculations have not been plotted in this case, since for $\zeta_{3 a}$ her results are very close to the present ones.

Note added in proof. There is a mistake in the $\zeta_{3 d}$ 's given in the fuller paper (Jones, 1970) resulting in an apparent disagreement between Figure 4 of this paper and the corresponding figure of Jones (1970).

\section{References}

Blume, M. and Watson, R. E.: 1962, Proc. Roy. Soc. A270, 127.

Condon, E. U. and Odabasi, H.: 1966, Spin-Orbit Interaction in Self-Consistent Fields, JILA rept. No. 61, University of Colorado.

Eissner, W. and Nussbaumer, H.: 1969, J. Phys. B. (2), 2, 1028. 
Froese, C.: 1967, Can. J. Phys. 45, 1501.

Godfredsen, E.: 1966, Astrophys. J. 145, 308.

Jones, M.: 1970, J. Phys. B. 3, 1571.

Layzer, D.: 1959, Ann. Phys. 8, 271.

Moore, C. E.: 1949, Atomic Energy Levels, Nat. Bur. Stand. Circ. No. 467 U.S. Govt. Printing Office, Washington. 